\title{
Long-term valacyclovir treatment and immune modulation for Herpes-associated erythema multiforme
}

\author{
JURATE STAIKUNIENE ${ }^{1}$, JURATE STANEVICIUTE ${ }^{2}$ \\ ${ }^{1}$ Department of Pulmonology and Immunology, Lithuanian University of Health Sciences, Kaunas, Lithuania \\ ${ }^{2}$ Department of Histology and Embryology, Lithuanian University of Health Sciences, Kaunas, Lithuania
}

\begin{abstract}
Objective: Erythema multiforme (EM) is an immune-mediated condition characterized by the appearance of target-like lesions on the skin and often accompanied by erosions or bullae involving the oral, genital, and/or ocular mucosae. $70 \%$ of recurrent EM cases are associated with HSV reactivation and it is labelled as herpes-associated erythema multiforme (HAEM). Recurrences are seen in approximately 20-25\% of EM cases and managing these conditions are challenging for both the patient and the doctor. The effectiveness of antiviral drugs is proven for Herpes simplex infection, however most patients use a multiplicity of alternative and complementary therapies.

Clinical presentation: We present clinical data of 3 patients with recurrent HAEM managed by long-term valacyclovir therapy and immunostimulation with Echinacea or replacement immunoglobulin therapy in the case of IgG1 subclass deficiency. The presented cases have demonstrated that immune mechanisms are relevant for HAEM recurrences.

Conclusions: The immune abnormalities, such as antibody deficiency, in the patients with HSV-associated EM can lead to frequent relapses of disease and should be evaluated. Long-term antiviral therapy with immunomodulation can control the relapses of HAEM.
\end{abstract}

Key words: erythema multiforme, herpes-associated erythema multiforme, HAEM, HSV infection.

(Cent Eur J Immunol 2015; 40 (3): 387-390)

\section{Introduction}

Erythema multiforme (EM) is an acute immune-mediated condition characterized by the appearance of target-like lesions on the skin and often accompanied by erosions or bullae involving the oral, genital, and/or ocular mucosae. Aetiology of EM may vary, but Herpes simplex virus (HSV) association has been identified in up to $70 \%$ of cases. Periodic reactivations of HSV induce frequent recurrences of erythema multiforme which is labelled herpes-associated erythema multiforme (HAEM). Recurrences are seen in approximately $20-25 \%$ of HAEM cases and patients may experience 2-24 episodes a year, an average of 6 attacks annually, with each episode lasting nearly 2 weeks for a mean duration of 9.5 years [2]. The effectiveness of antiviral drugs is proven for HSV infection, however most patients use a multiplicity of alternative and complementary therapies.

We present clinical data of HAEM which strongly suggest that immune mechanisms are relevant for HAEM recurrences and a combination of antiviral drugs and immunostimulation with Echinacea and replacement immu- noglobulin therapy in antibody deficiency can effectively control HAEM. Our article reviews scientific data of traditional disease management strategies and Echinacea plant immunomodulating properties.

\section{Clinical presentation}

All the patients presented in this case report had signed a patient's consent form.

Three patients ( 2 females aged 53 and 39 yrs., and 1 man aged 42 yrs.) had the history of frequent (every month) recurrent orofacial Herpes simplex and later on presented EM with typical target lesions in skin and multiple ulcers in the oral and genital mucosae. Erythema multiforme was confirmed by typical skin and mucosal lesions (Figs. 1, 2) and histology of skin biopsy (Figs. 3, 4). The immunological status was evaluated by assessing the serum immunoglobulin level, lymphocyte phenotyping, nitro blue tetrazolium test (NBT), antibodies against HIV and HSV infections. All patients were HIV negative and strongly positive to HSV (Table 1). Other possible caus-

Correspondence: Jurate Staneviciute, Department of Pulmonology and Immunology, Lithuanian University of Health Sciences, Eiveniu 2, LT 50009 Kaunas, Lithuania 
ative EM agents, such as drugs, have been ruled out. One of the patients had a substantially low IgG1 level and was diagnosed with a selective IgG1 subclass deficiency and was treated with intravenous immunoglobulin replacement therapy (total dose $2 \mathrm{~g} / \mathrm{kg}$ ) (Table 2). Other patients had no abnormalities in lab tests. All patients were treated with a prolonged course of the antiviral drug (valacyclovir) and immunostimulation with intramuscular Echinacea 2-3 times a week for 2 months. The initial dosage for valacyclovir was $500 \mathrm{mg}$ twice for 5-10 days, later the dosage

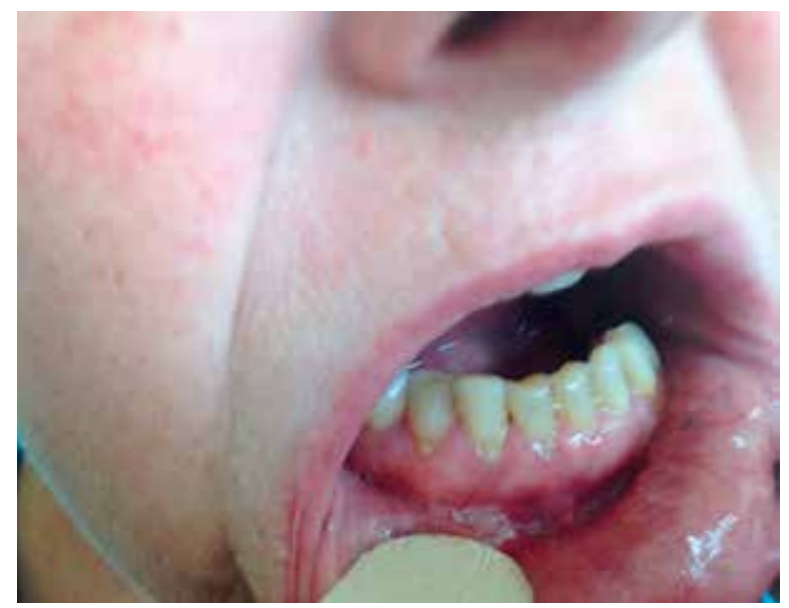

Fig. 1. Oral ulcers in erythema multiforme

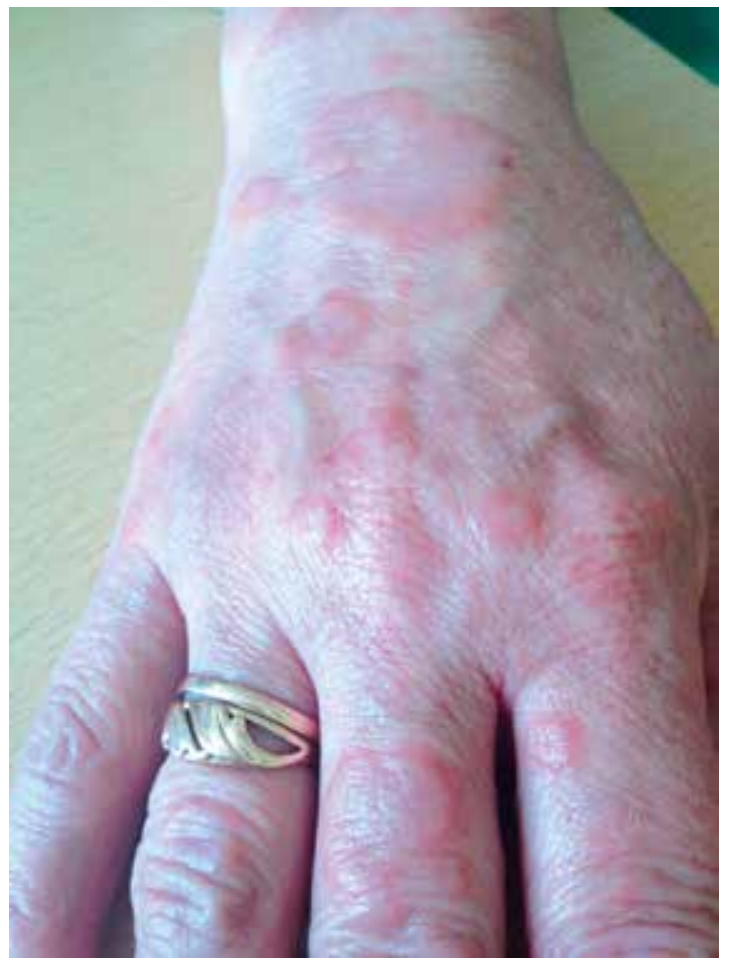

Fig. 2. Typical skin lesions in hands was reduced to $500 \mathrm{mg}$ daily, and being free of recurrence after 3-4 months - twice a week for 2 months and eventually was discontinued. Drug safety has been assessed regularly by a set of laboratory tests: total blood count, serum creatinine, urea, and liver enzymes. All the tests were within normal range and no side effects were observed. On one-year follow up patients did not present HAEM.

\section{Discussion}

Herpes simplex virus infection is extremely common in humans; it is estimated that $70-90 \%$ of people over the age of 18 have antibodies to both types - HSV-1 and/or HSV-2 and carry the latent virus, with approximately $25 \%$ showing clinical symptoms $[1,2]$. HSV can occur at diverse sites with a wide range of symptoms and can implicate erythema multiforme. Aetiology of EM may vary, but Herpes simplex virus association has been identified in up to $70 \%$ cases and some studies have estimated that $61 \%$ to $100 \%$ of recurrent EM cases are due to HSV [1, 3, 4].

The immune system employs a variety of strategies to eliminate the virus, whereas the virus has developed immune evasion mechanisms to escape its elimination. DNA viruses such as Herpes viruses utilize diverse mechanisms to extend the time-window for viral replication and spreading of virus particles and are capable of interfering with the host's immune system at almost every level of immune clearance. Viral evasion molecules

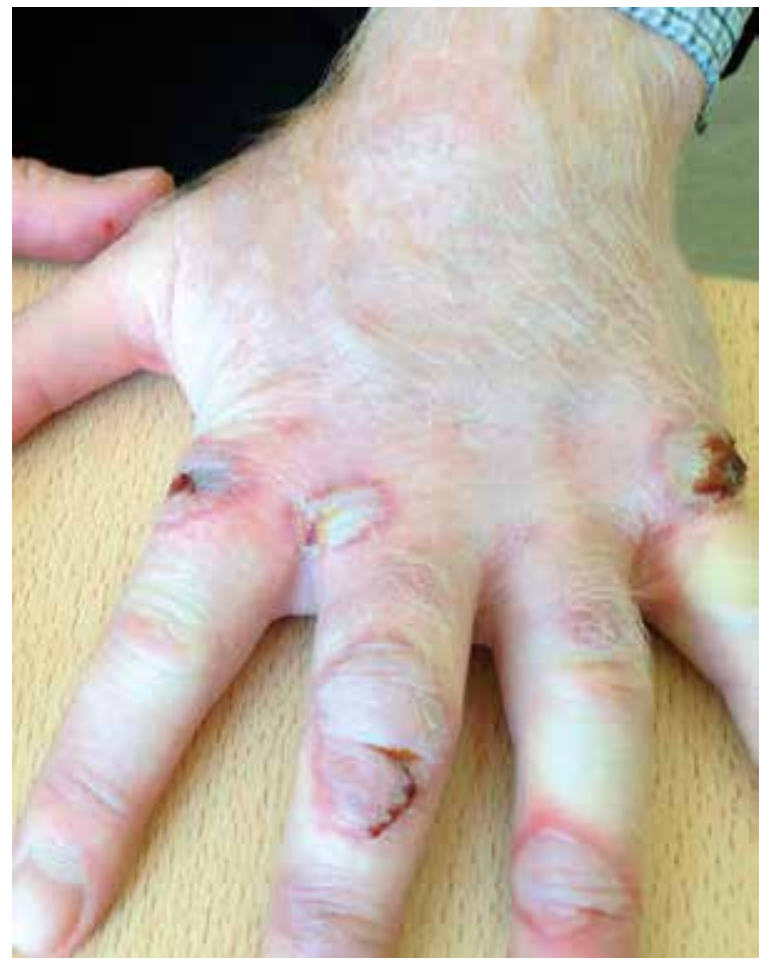




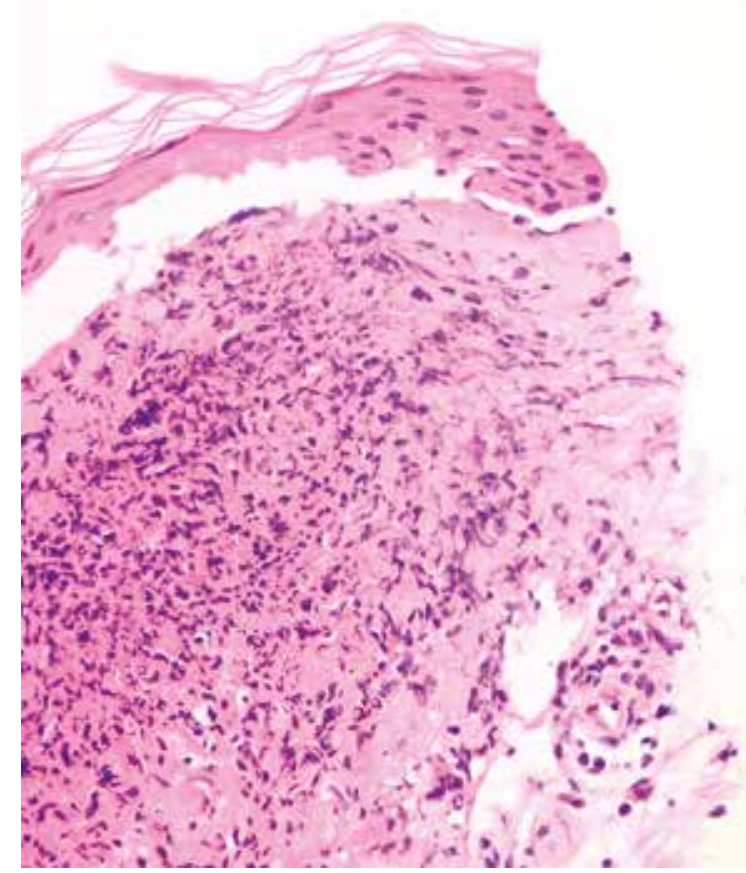

Fig. 3. Epidermal keratinocyte necrosis in desquamated epidermal fragment

target components of innate and acquired immunity, including complement proteins, natural killer cells, MHC Class I or Class II molecules and antibody [5]. After the initial infection, HSV replicates in the skin or mucosal epithelium, then infects sensory nerves and is transported to the involved sensory ganglia. Here HSV establishes a life-long latent infection that is punctuated by periodic reactivations and may induce recurrence of EM [1]. It is essential for the immune system to induce an adequate response for clearance of the virus in order to break the vicious circle of the latency and recurrence of the virus

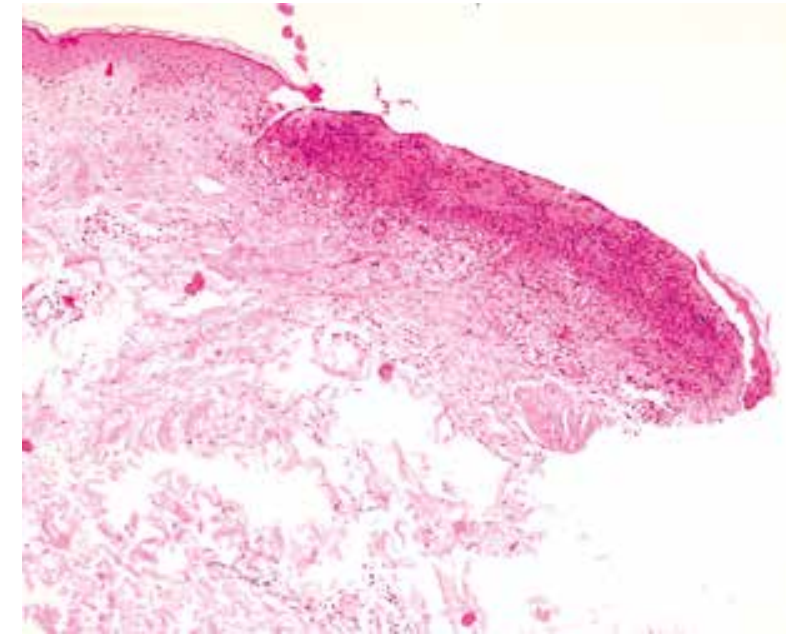

Fig. 4. The accumulation of inflammatory cells (lymphocytes, neutrophils, eosinophils) at the bottom of ulceration accumulation in limited lesion

and to develop antibodies to fight the virus. The presence of IgM herpes simplex virus HSV antibodies indicates an acute infection and IgG-class antibody indicates previous exposure. In our presented clinical course overview, the immune system was evaluated for all patients, all of them had elevated IgM and IgG levels indicating virus reactivation. Reasonably the patients had undergone long-term valacyclovir therapy to suppress the virus replication. One of the patients presented extremely high antibodies of IgG to HSV compared to others. The high titres of IgG may be due to high virus replication or to the immune system abnormality: either antibody activity to defined viral antigens or viral neutralization disorder. One of the patients with extremely high IgG to HSV was diagnosed with a selective $\mathrm{IgG} 1$ subclass deficiency. The presented clinical course strengthens the importance of immune response

Table 1. The presence of IgM, IgG antibodies to HSV before and after the courses of antiviral drugs and immune stimulation

\begin{tabular}{ccccc}
\hline \multirow{2}{*}{ Patients } & \multicolumn{2}{c}{ Anti-HSV level before treatment (U/ml) } & \multicolumn{2}{c}{ Anti-HSV level after treatment (U/ml) } \\
\cline { 2 - 5 } & $\mathrm{IgG}$ & $\mathrm{IgM}$ & $\mathrm{IgG}$ & $\mathrm{IgM}$ \\
\hline No. 1 & 4038.9 & 44.9 & 783.3 & 27.6 \\
\hline No. 2 & 6833 & 750 & not done & not done \\
\hline No. 3 & 970000 & 30.2 & 3974 & negative \\
\hline
\end{tabular}

Table 2. Evaluation of humoral immune response before and after the intravenous immunoglobulin replacement therapy for the patient with a selective IgG1 subclass deficiency

\begin{tabular}{ccccc}
\hline & Serum IgG (g/l) & $\begin{array}{c}\text { Serum } \\
\text { IgG1 (g/l) }\end{array}$ & $\begin{array}{c}\text { Anti-HSV } \\
\text { IgM (U/ml) }\end{array}$ & $\begin{array}{c}\text { Anti-HSV IgG } \\
(\mathbf{U} / \mathbf{m l})\end{array}$ \\
\hline Before treatment & 6 & 3.12 & 30.2 & 970000 \\
\hline After treatment & 9.6 & 4.09 & negative & 3974 \\
\hline
\end{tabular}


evaluation for patients with recurrence of the disease as the possible underlying immunity abnormalities can be relevant to the HAEM relapses. IgG subclass deficiency is often undiagnosed for long periods of time and may be presented in any age group. It is important to stress that replacement of intravenous immunoglobulin leads to a successful disease control.

Managing HAEM can be challenging for both the patient and the doctor. Antiviral drugs are used to shorten the clinical course, prevent complications, decrease transmission, and eliminate established latency. Suppression of HSV can prevent HSV-associated erythema multiforme, but antiviral treatment started after the eruption of erythema multiforme has no effect on the course of the erythema multiforme. Prophylaxis for recurrence of HAEM should be considered in patients with more than 5 attacks per year $[6,7]$. Low-dose acyclovir (200 $\mathrm{mg}$ qd to $400 \mathrm{mg}$ bid) can be effective for recurrence of HAEM, even in the subclinical HSV infection [6, 7]. Prophylaxis may be required for 6-12 months or longer [8]. Our patients were safely treated with a prolonged course of valacyclovir, starting from $500 \mathrm{mg}$ twice for 5-10 days, later the dosage was reduced to $500 \mathrm{mg}$ daily, and being free of recurrence after 3-4 months - twice a week for 2 months and eventually was discontinued. The effectiveness of antiviral drugs is proven for Herpes simplex infection, but recurrences are seen in approximately $20-25 \%$ of EM cases and patients may experience 2-24 episodes a year, even with adequate antiviral treatment. It may be due to viral resistance toward antiviral treatment or the underlying immune mechanism which fails to define or neutralize viral antigens.

Because of the incidents of frequent recurrences most patients use a multiplicity of alternative therapies apart from standard antiviral drugs. Recently, an increased interest has been observed in natural medicines, such as Echinacea, that have immunotropic activity and which can increase cellular and humoral immunity against pathogens. Echinacea purpurea extract has been used in prophylaxis and therapy of various viral infections, mainly respiratory tract infections in animals and humans [9]. In vitro studies have shown that Echinacea acts directly on a number of cell types, including natural killer cells, polymorphonuclear leukocytes and macrophages, also have some effect on B cell proliferation [10]. The reviewed scientific data on Echinacea plant immunomodulating properties and the results of individual trials consistently show positive trends, although potential effects are of questionable clinical relevance. In our presented clinical course, the combination of prolonged courses of antiviral drugs and additional immunostimulation with Echinacea could enhance the efficiency of immune response leading to reduced Herpes simplex viral replication in the skin and mucosa and contributed to the control of HAEM.

In conclusion, the immune abnormalities, such as antibody deficiency, in the patients with HSV-associated
EM can lead to frequent relapses of disease and should be evaluated and replacement therapy should be initiated. The long-term treatment with valacyclovir and immunostimulation with Echinacea proved to be effective and safe in our patients. These results support the concept of using long-term valacyclovir and Echinacea as additional medicine for the treatment of Herpes simplex-associated EM, but clinical data are insufficient to be confident about the efficacy and safety. Further clinical investigations are needed to be conducted with adequate accuracy to provide guidance to the patients and doctors using these products.

The authors declare no conflict of interest.

\section{References}

1. Fatahzadeh M, Schwartz RA (2007): Human herpes simplex virus infetions: epidemiology, pathogenesis, symptomatology, diagnosis, and management. J Am Acad Dermatol 57: 737763.

2. Osterne RL, Matos Brito RG, Pacheco IA, et al. (2009): Management of erythema multiforme associated with recurrent herpes infection: a case report. J Can Dent Assoc 75: 597-601.

3. Arduino PG, Porter SR (2008): Herpes Simplex Virus Type 1 infection: overview on relevant clinico-pathological features. J Oral Pathol Med 37: 107-121.

4. Farthing P, Bagan JV, Scully C (2005): Mucosal disease series. Number IV. Erythema multiforme. Oral Dis 11: 261-267.

5. Vossen MT, Westerhout EM, Söderberg-Nauclér C, Wiertz EJ. (2002): Viral immune evasion: a masterpiece of evolution. Immunogenetics 54: 527-542.

6. Huff JC (1988): Acyclovir for recurrent erythema multiforme caused by herpes simplex. J Am Acad Dermatol 18 (1 Pt 2): 197-199.

7. Tatnall FM, Schofield JK, Leigh IM (1995): A double-blind, placebo-controlled trial of continuous acyclovir therapy in recurrent erythema multiforme. Br J Dermatol 132: 267-270.

8. Kerob D, Assier-Bonnet H, Esnault-Gelly P, et al. (1998): Recurrent erythema multiforme unresponsive to acyclovir prophylaxis and responsive to valacyclovir continuous therapy. Arch Dermatol 134: 876-877.

9. Mishima S, Saito K, Maruyama H, et al. (2004): Antioxidant and immuno-enhancing effects of Echinacea purpurea. Biol Pharm Bull 27: 1004-1009.

10. Barrett BP, Brown RL, Locken K, et al. (2002): Treatment of the common cold with unrefined echinacea. A randomized, double-blind, placebo-controlled trial. Ann Intern Med 137: 939-946. 